APPENDICE IV A

\title{
UN ANOMOTAENIA A PORES GÉNITAUX UNILATÉRAUX CHEZ TRINGA OCHROPUS L. 1758
}

\author{
Par Robert-Ph. DOLLFUS
}

J'ai récolté 16 individus de ce Cestode (fig. 126-130) dans l'intestin d'un Tringa ochropus L., à Richelieu (Indre-et-Loire).

Description : Longueur: $55-60 \mathrm{~mm}$., avec une plus grande largeur de$2 \mathrm{~mm}$.; scolex long (sans le rostre) de 0,22-0,25 jusqu'au niveau du bord postérieur des ventouses, large de 0,42 . Cou (entre le niveau du bord postérieur des ventouses et le début de la segmentation) long de 0,40 , largede 0,39 .

La partie évaginée du rostre est longue de 0,08 à 0,12 , avec un diamètre de 0,10 à 0,16 ; elle porte une couronne de 32 crochets longs d'environ $40 \mu$, ces crochets ne sont pas régulièrement partout sur deux rangs, de sorte qu'il n'est pas certain que l'on doive considérer cette couronne comme double. Les ventouses, inermes, ont un diamètre variant de 0,12-0,14 à 0,18-0,20.

Premiers proglottis plus larges que longs (0,04 de long sur 0,30 de large), puis plus longs que larges (0,50 de long sur 0,30 de large), ensuite carrés, ensuite plus larges que longs dans les deux derniers tiers du strobile. Il y a 75-120 proglottis environ; ils sont craspédotes. Les pores génitaux sont unilatéraux, très rapprochés de l'angle antérieur du segment. Il y a 44-52 testicules, en un seul groupe occupant la partie postérieure du segment et ne dépassant pas latéralement le canal excréteur ventral; leur diamètre atteint $84 \mu$ environ. Le canal déférent s'étend transversalement sur un peu plus d'un tiers de la largeur du segment et ne forme pas de vésicule séminale. La poche du cirre est extrêmement réduite (environ $40 \times 20 \mu$ ); le cirre, piriforme, est fortement épineux, il peut atteindre, en évagination complète, $96 \mu$ de long. Le vagin s'étend transversalement au-dessous du canal déférent et, vers le milieu de la largeur du segment, se dilate en un réceptacle séminal ovale allongé, mesurant de $140 \times 75 \mu$ à $180 \times 100 \mu$. L'ovaire, lobé, très petit, ne dépasse pas $240 \mu$ transversalement et $120 \mu$. 
longitudinalement; dans les préparations in toto, la délimitation entre l'ovaire et le vitellogène est mal distincte.

L'utérus apparaît nettement vers le $90^{\circ}$ segment, sous forme d'une masselobée près de la limite antérieure du segment, dans la moitié antiporale; cette masse s'étend progressivement vers le côté poral et en direction postérieure vers les testicules; elle occupe bientôt tout le parenchyme médullaire, puis pénètre plus ou moins latéralement dans le parenchyme cortical lorsque le segment devient gravide.

L'utérus est persistant, mais sa cavité n'est pas libre, elle paraît remplie: par un lacis serré de petits tubes très fins où les œufs sont disposés à la file, les uns à la suite des autres, en chapelets. L'oncosphère mesure environ: $30 \times 16 \mu$, sa coque $43 \times 25 \mu$, les crochets ont une longueur de 11,5-12 $\mu$.

Discussion : Chez ce Charadriiforme, huit espèces de Cestodes sont connues, d'après Ch. Joyeux et J. G. Baer (1936, p. 570) ; aucune d'entre elles ne correspond à l'espèce ci-dessus. Sur les huit espèces, quatre ont les pores sexuels unilatéraux: deux espèces d'Haploparaxis et deux espèces de Dilepis. Notre Cestode, avec ses 32 crochets rostraux et ses 50 testicules, ne s'apparente pas aux Haploparaxis; il n'est pas référable à Dilepis limosa O. Fuhrmann 1907 dont les crochets rostraux, au nombre de 20, mesurent 110 et $99 \mu$, non plus à Dilepis ochropodis Neslobinsky 1911, dont les. crochets rostraux, à peu près de même longueur $(43 \mu)$, ont une forme complètement différente. Parmi les 75 espèces de Cestodes de Charadriiformes mentionnés dans la « Faune de France », comme ayant été trouvées, en France ou hors de France, chez des Oiseaux existant dans la faune française, aucune ne correspond exactement à notre espèce, mais celles qui s'en rapprochent le plus sont des Anomotaenia.

Chez les Anomotaenia, les pores génitaux ne sont pas unilatéraux; si donc notre Cestode est un Anomotaenia, il est une exception dans ce genre. On connaît trois espèces d'Anomotaenia chez Tringa ochropus L., ce sont globulus (Wedl), citrus (Krabbe), arionis (Siebold).

Chez citrus, il y a 20-25 crochets mesurant 54-62 $\mu$ pour ceux de la couronne antérieure et 51-56 $\mu$ pour ceux de la couronne postérieure ; chez arionis, il y a 20 crochets de $32-36 \mu$; chez globulus, il y a 30 crochets de38-40 $\mu$, nous avons donc comparé notre espèce à globulus (1).

Sous le nom « Taenia Globulus (nov. spec.) », Carl Wedl (1855, p. 9-10, 25, pl. I, fig. 8-9 b) a décrit un Anomotaenia, d'après des individus trouvés: chez un Scolopax gallinula L. 1766 de la rivière Theiss (Hongrie); il y a deux couronnes de chacune 14-16 crochets, dont la longueur n'est pas. indiquée. D'après la description et la figure, le cirre n'est pas épineux.

A la même espèce, Harald Krabbe (1869, p. 271, 366, pl. III, fig. 53-54 b crochets, fig. 55 œuf) a rapporté un spécimen long de $15 \mathrm{~mm}$., trouvé chez

(1) Quelques auteurs ont écrit « globula \$! affreux barbarisme. 

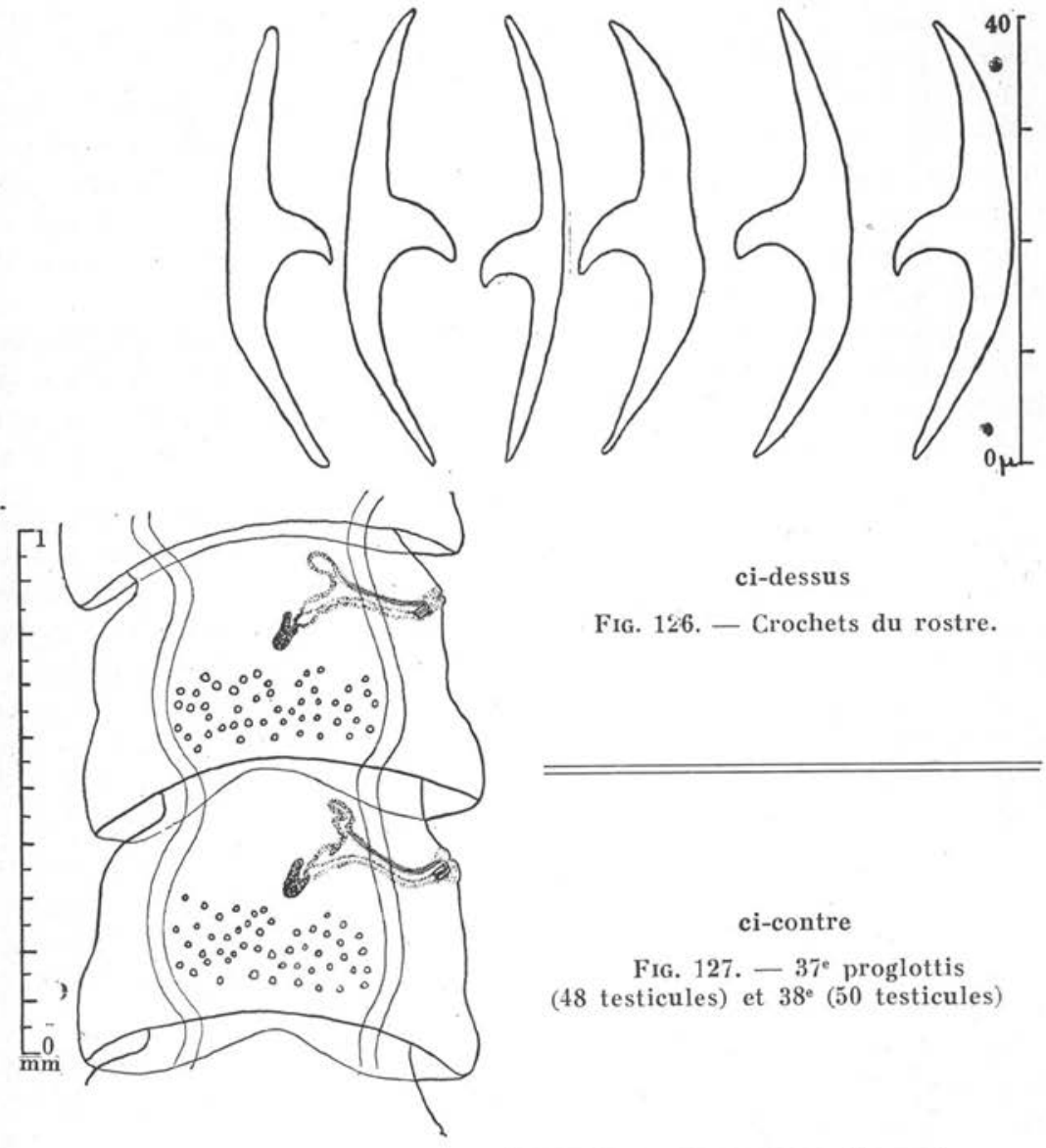

ci-contre

FIG. 126. - Crochets du rostre.
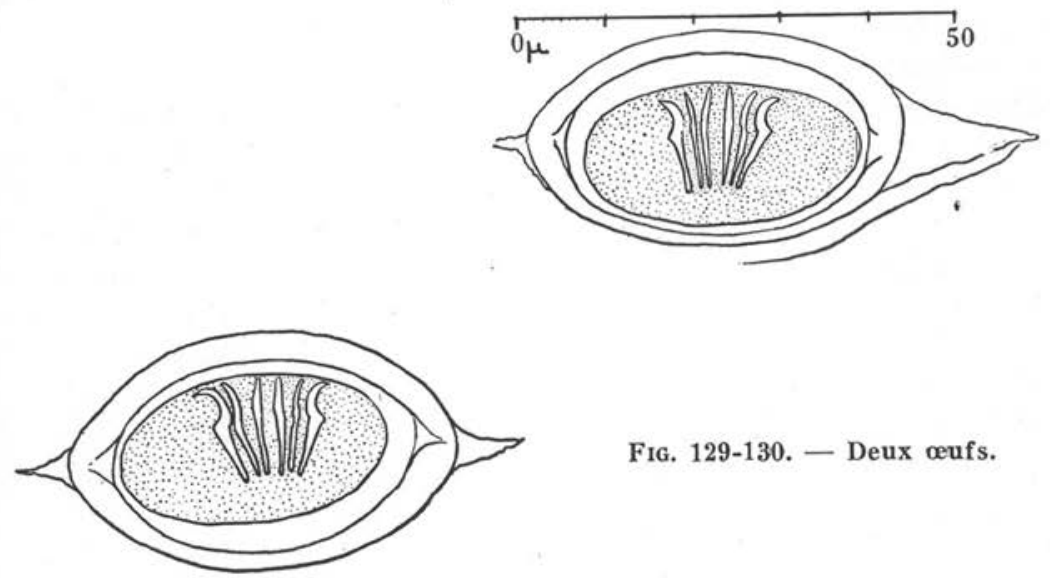

FIG. 127. $-37^{\mathrm{e}}$ proglottis (48 testicules) et $38^{\circ}$ ( 50 testicules)

Fig. 129-130. - Deux œufs. 


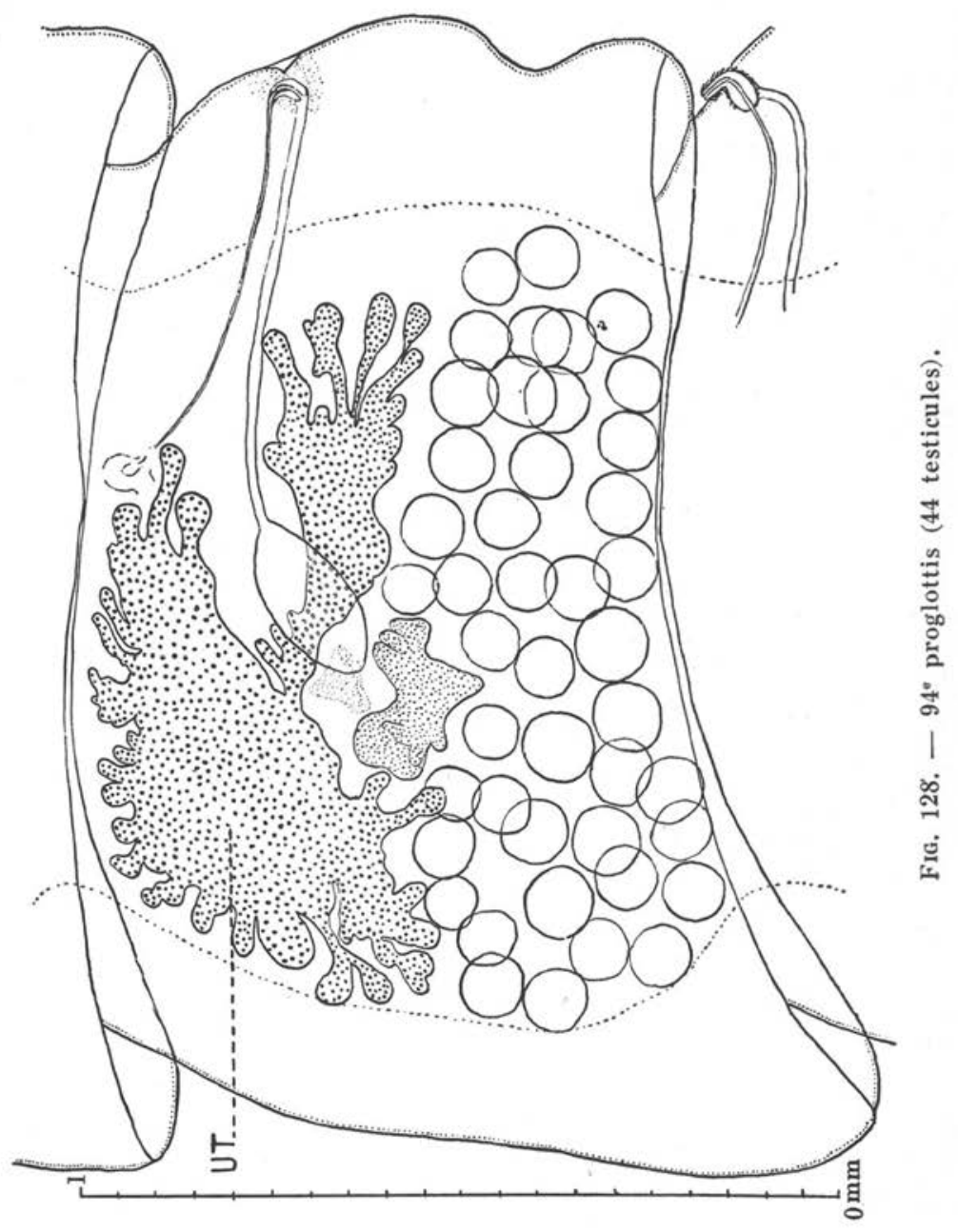


Tringa ochropus L. en Egypte, par Bilharz. Krabbe a compté 30 crochets de $38 \mu$; ces crochets ont une garde de forme différente de celle des crochets de mes spécimens de Richelieu.

Wl. Clerc $(1902$, p. $661 ; 1903$, p. 323-325, pl. IX, fig. 46) a décrit comme «Choanotaenia globulus Wedl » un Cestode de Tringa ochropus L. et Tringa pugnax L. de l'Oural; les crochets (nombre?) ont 38-40 $\mu$. L'ovaire est à deux ailes très ramifiées, dendritiques. Il n'est pas question d'un cirre épineux. Anatomiquement, mes spécimens ne correspondent pas à la description de Clerc.

Je laisse provisoirement en suspens l'identification de mes spécimens du Chevalier cul-blanc de Richelieu.

\section{OUVRAGES CITÉS}

Clerc (Wladimir), 1902. - Contribution à l'étude de la faune helminthologique de l'Oural. Communication préliminaire, II. Zoolog. Anzeiger, XXV, $\mathrm{n}^{\circ} 681,15-9-1902$, p. 658-664, fig. 1-5.

- 1903. - Contribution à l'étude de la faune helminthologique de l'Oural. Revue Suisse de Zoologie, t. XI, 5-9-1903, p. 241-368, fig., texte 1-6, pl. VIII-XI, fig. 1-89.

Joyeux (Charles) et BAER (Jean-G.), 1936. - Cestodes. Faune de France, XXX, Paris, 1936, 613 p., 569 fig.

Krabbe (Harald), 1869. - Bidrag til Kundskab om Fuglenes Baendelorme. Kongl. Danske Videnskab. Selskabs Skrifter, naturv. og math. Afd., 5R, vol. VIII, n 6,1869 , p. 249-363 (résumé en français, p. 364-368), pl. I-X, fig. 1-303.

WEDL (Carl), 1855. - Charakteristik mehrerer grösstentheils neuer Tänien. Sitzungsber. der Kais. Akademie der Wissenschaften Wien, mathem. naturwiss. Classe, Bd. XVIII, n ${ }^{\circ}$, nov. 1855 , p. 5-27, pl. I-III, fig. $1-43$. 